\title{
Digestive Endoscopy
}

\section{Biliary sphincterotomy reduces the risk of acute gallstone pancreatitis recurrence in non-candidates for cholecystectomy ${ }^{\text {is }}$}

\author{
Irene García de la Filia Molina ${ }^{a, b, 1}$, Ana García García de Paredes a,b,c,*,1, \\ Antonio Martínez Ortega ${ }^{a, b}$, Natalia Marcos Carrasco ${ }^{a, b}$, \\ Enrique Rodríguez De Santiago ${ }^{a, b, c}$, Rubén Sánchez Aldehuelo a,b, \\ Jose Ramón Foruny Olcina a,b,c, Juan Ángel González Martin a,b,c, Sergio López Duran a,b, \\ Enrique Vázquez Sequeiros ${ }^{\mathrm{a}, \mathrm{b}, \mathrm{c}}$, Agustín Albillos ${ }^{\mathrm{a}, \mathrm{b}, \mathrm{c}, \mathrm{d}}$ \\ a Gastroenterology and Hepatology Department, Ramón y Cajal University Hospital, Madrid, Spain \\ b University of Alcalá, Madrid, Spain \\ ${ }^{\mathrm{c}}$ Ramón y Cajal Institute of Biosanitary Research (IRYCIS), Madrid, Spain \\ d Biomedical Research Center in Liver and Digestive Diseases Network (CIBERehd), Carlos III Health Institute, Madrid, Spain
}

\section{A R T I C L E I N F O}

\section{Article history:}

\section{Keywords:}

Biliary sphincterotomy

Endoscopic retrograde

cholangiopancreatography

Gallstones

Pancreatitis

Recurrence

\begin{abstract}
A B S T R A C T
Background: Population aging and comorbidity are leading to an increase in patients unfit for cholecystectomy.

Aims: To evaluate whether endoscopic biliary sphincterotomy after a first episode of acute gallstone pancreatitis reduces the risk of pancreatitis recurrence and gallstone-related events in non-surgical candidates.

Methods: Retrospective study of patients admitted for a first episode of acute gallstone pancreatitis rejected for cholecystectomy between 2013-2018. The role of endoscopic sphincterotomy was evaluated by adjusting for age, severity of pancreatitis, and presence of choledocholithiasis.

Results: We included 247 patients (mean age $80 \pm 12$ years; Charlson index: 5 ; severity of pancreatitis: $72 \%$ mild). Sphincterotomy was performed in $23.9 \%$. Recurrence of pancreatitis occurred in $17.4 \%$ patients (median follow-up: 426 days). The one-year cumulative incidence of a new episode of pancreatitis was $1.8 \%$ (95\% confidence interval $[\mathrm{CI}]: 0.2-12 \%)$ and $23 \%(95 \% \mathrm{CI}: 17-31 \%)$ in patients with and without sphincterotomy, respectively $(\mathrm{p}=0.006)$. In multivariate analysis, sphincterotomy showed a protective role for recurrence of pancreatitis (adjusted hazard ratio [HR]: $0.29,95 \% \mathrm{CI}: 0.08-0.92, \mathrm{p}=0.037$ ) and for any gallstone-related event (HR 0.46, 95\% CI: 0.21-0.98, $\mathrm{p}=0.043$ ).

Conclusions: Endoscopic biliary sphincterotomy reduced the risk of gallstone pancreatitis recurrence and other biliary-related disorders in patients with a first episode of pancreatitis non-candidates for cholecystectomy.
\end{abstract}

(C) 2019 Editrice Gastroenterologica Italiana S.r.l. Published by Elsevier Ltd. All rights reserved.

\section{Introduction}

Acute pancreatitis is a common gastrointestinal condition with a growing incidence (13-45 cases per 100,000 persons/year) [1] and an estimated mortality that rises to $30 \%$ in severe cases [2].

\footnotetext{
is Presented as an abstract at 40 Congreso Sociedad Española de Endoscopia Digestiva (SEED), Zaragoza, Spain, November 2018.

* Corresponding author at: Gastroenterology and Hepatology Department, Hospital Universitario Ramón y Cajal, University of Alcalá, Madrid, Spain.

E-mail address: anaggparedes@gmail.com (A. García García de Paredes).

1 Irene García de la Filia and Ana García García de Paredes share co-first authorship.
}

Gallstone pancreatitis is caused by migration of stones from the gallbladder through the ampulla of Vater where they can cause transitory blockage of the pancreatic duct [3], and this is the most frequent etiology accounting for $40-70 \%$ of the cases [4].

After the first episode of acute gallstone pancreatitis (AGP) the risk of recurrence is notably high, ranging between $2.5 \%$ and $63 \%$ [5-7]. Recurrent episodes entail high mortality and morbidity rates (10\% and $40 \%$ respectively) [8]. Therefore, it is necessary to carry out preventive measures addressed to decrease the risk of recurrence. Cholecystectomy is currently considered the definitive treatment for AGP as it has shown to reduce not only the risk of pancreatitis (down to 1-1.7\%) but also the recurrence of other gallstone-related disorders such as cholecystitis, cholangitis and choledocholithia- 
sis [9-14]. Accordingly, cholecystectomy is widely recommended after the first episode of AGP to prevent further attacks, and it should be performed before hospital discharge due to the fact that most recurrences occur within the first month $[2,15,16]$. Despite being the gold standard treatment, cholecystectomy is not always an option as it is often rejected due to advanced age or major comorbidities. Unfortunately, this situation is becoming more frequent in clinical practice as a result of the increase in life expectancy and a higher incidence of gallstone-related disease in the elderly. Therefore, there is an urgent need to explore alternative prophylactic approaches to reduce the risk of recurrence of AGP in those unfit to undergo cholecystectomy.

The benefit of endoscopic retrograde cholangiopancreatography (ERCP) in this setting remains unclear. Some authors have suggested that endoscopic biliary sphincterotomy (EBS) may be effective to prevent AGP recurrence based on the hypothesis that a wide-open sphincter may facilitate the passage of stones through the papilla, thus reducing the risk of obstruction [17-20]. Based on these data, some guidelines suggest that ERCP with EBS may be performed in patients who cannot undergo elective cholecystectomy because of high surgical risk $[2,16,21]$. However, studies aimed to evaluate the protective role of EBS in the recurrence of AGP are scarce and often limited by a small number of patients, a short follow-up, a lack of control group or an administrative database source of data $[4,17,22]$.

This study was designed to assess the benefit of EBS to reduce the risk of recurrence of AGP when cholecystectomy is not an option. Secondarily, readmissions for biliary-related events other than pancreatitis were evaluated.

\section{Patients and methods}

This was a retrospective cohort study conducted at a tertiary academic hospital (Hospital Universitario Ramón y Cajal, Madrid, Spain). The study protocol adhered to the principles of the Declaration of Helsinki and was approved by the Ethics Committee of the Hospital Universitario Ramón y Cajal. The Ethics Committee accepted exemption of individual informed consent for the inclusion in the study due to its retrospective design.

\subsection{Study population}

Hospital and primary health care clinical records of all patients admitted for acute pancreatitis between January 2013 and January 2018 were reviewed. Patients with a first episode of AGP during the study period who did not undergo cholecystectomy due to high surgical risk were included for analysis. Patients were considered unfit for surgery according to the physician in charge. Exclusion criteria were: (1) previous episodes of AGP, (2) prior cholecystectomy, and (3) cholecystectomy after the first episode of AGP.

\subsection{Definitions}

The diagnosis of acute pancreatitis was made according to the International Association of Pancreatology and American Pancreatic Association criteria [2], and it was defined as fulfillment of 2 out of 3 of the following criteria: (1) clinical (upper abdominal pain); (2) laboratory (serum amylase or lipase three times greater than normal); (3) radiological evidence of pancreatitis (CT, MRI, ultrasonography). Biliary etiology was based on demonstration of cholelithiasis or choledocholithiasis on image test and exclusion of other causes of pancreatitis (alcoholism, toxic, hypercalcemia, hypertriglyceridemia, chronic pancreatitis and pancreatic neoplasms). Pancreatitis severity was graded according to the Revised Atlanta Classification into the following categories: mild (absence of organ failure or local or systemic complications), moderate (transient organic failure $<48 \mathrm{~h}$ or presence of local complications) or severe (persistent organ failure $>48 \mathrm{~h}$ ) [23]. Comorbidity was assessed by the American Society of Anesthesiologists (ASA) classification and the Charlson index. Recurrent pancreatitis was diagnosed if the patient was readmitted for a new episode of AGP, as defined above.

\subsection{ERCP procedure}

Patients were divided into two different groups based on the performance of ERCP with EBS. ERCP outcomes were retrieved from a prospectively collected database. ERCP was performed in a standard manner, either urgently, during the index admission, or electively after hospital discharge according to the criterion of the physician in charge. ERCP was performed by four experienced endoscopists and written informed consent was obtained in all cases. Adverse events were defined according to the American Society for Gastrointestinal Endoscopy (ASGE) lexicon criteria [24].

\subsection{Follow-up}

Time to AGP and other biliary-related disorders recurrence was recorded. Long-term outcomes between the date of discharge and readmission for gallstone-related disease or until the end of the follow up in the absence of recurrence were compared.

\subsection{Study endpoints}

The primary endpoint was to assess the effectiveness of EBS to reduce the risk of recurrence of AGP in patients considered unfit for cholecystectomy. As secondary endpoint, we evaluated the role of EBS to prevent other biliary events (cholecystitis, choledocholithiasis, and cholangitis) after a first episode of AGP in patients not subsidiary for cholecystectomy.

\subsection{Statistical analysis}

Continuous variables were reported as mean \pm standard deviation (SD) when normality was met and as median and interquartile range (IQR) for non-normally distributed variables. The Shapiro-Wilk test and distributional plots were used to assess normality. T-Student test was used to compare differences between groups for normally distributed variables, and the U Mann-Whitney test for non-normally distributed variables. Categorical variables were described as absolute and relative frequencies, and $\chi^{2}$ test (or Fisher exact test when necessary) were used for intergroup comparisons. We plotted the cumulative incidence of recurrence of AGP and readmission for other biliary-related events by using the Kaplan-Meier survival function estimates. Patients who died before readmission were rightcensored at the time of death. A multivariate Cox proportional hazards regression was used to evaluate the role of EBS on readmissions for AGP and other gallstone-related events. Cox regression results were expressed using adjusted Hazard Ratio (HR) and a 95\% confident interval $(\mathrm{CI})$. The role of EBS was evaluated by adjusting for age, treatment with ursodeoxycholic acid (UDCA), severity of pancreatitis and presence of choledocholithiasis. Proportionalhazards assumptions were tested via Schoenfeld residuals. The number of EBS needed to prevent one AGP was calculated according to Altman et al. [25]. All analyses were two-tailed, and significance was set at $\mathrm{p}<0.05$. All statistical analyses were performed using Stata/IC 14.0. 


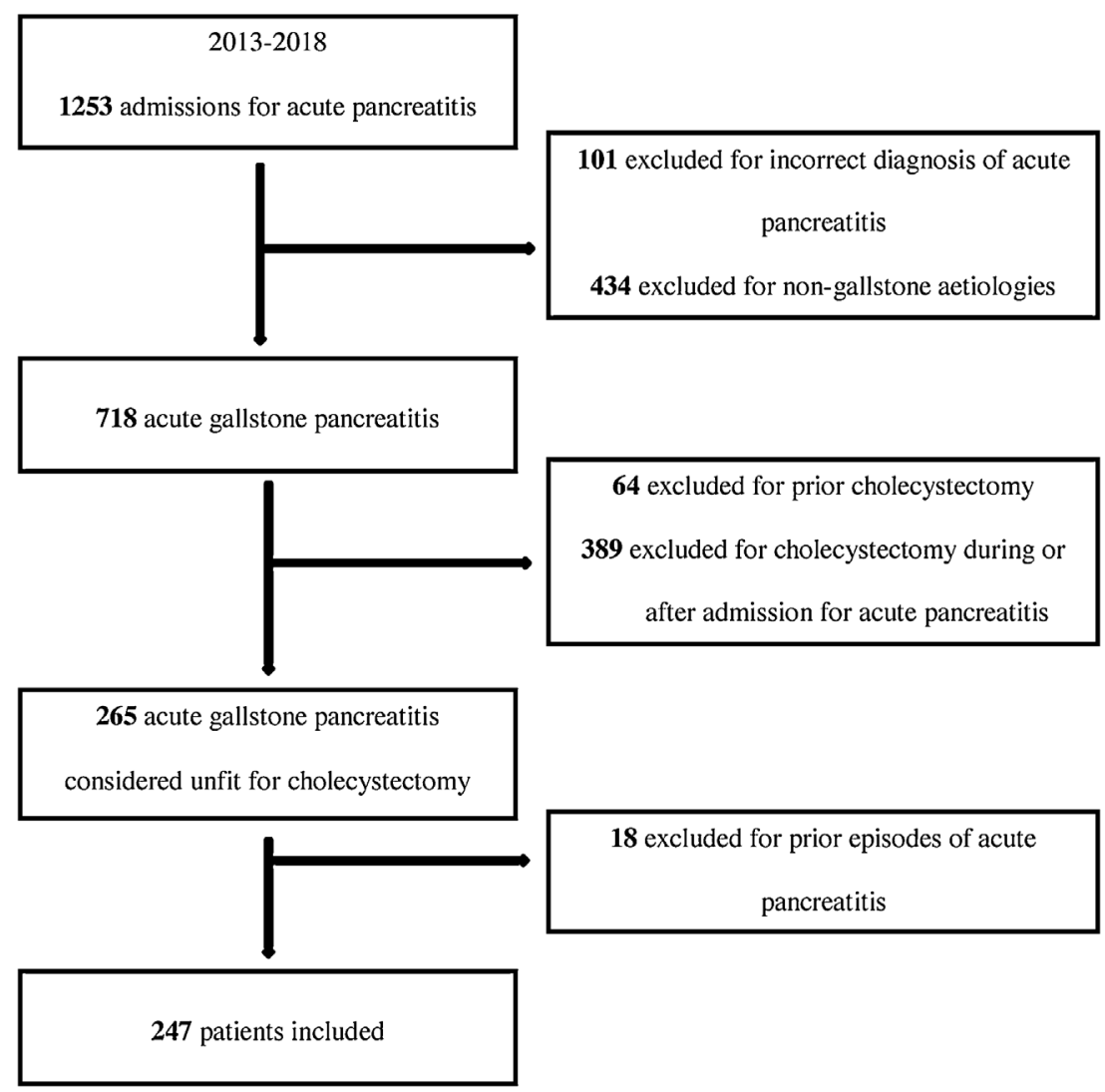

Fig. 1. Study flow chart.

\section{Results}

We identified a study sample of 247 patients (59\% female; mean age $80 \pm 12$ years) admitted for a first episode of AGP during the study period in which cholecystectomy was rejected due to high surgical risk (Fig. 1).

\subsection{Index admission}

\subsubsection{Patient characteristics}

Table 1 summarizes the clinical characteristics of the study population. At admission, 109 (84.6\%) patients had altered hepatic biochemistry and in 97 (39.2\%) patients choledocholithiasis was identified on image tests.

\subsubsection{ERCP procedure}

During first admission 188 (76.2\%) patients had conservative management and 59 (23.8\%) underwent ERCP for the following indications: 42 for confirmed or suspected choledocholithiasis (71.2\%), 15 for cholangitis (25.4\%), and 2 for preventive intention (3.4\%). ERCP was performed during admission in 46 patients ( $78 \%$ ) and in 13 (22\%) after hospital discharge. EBS was performed in 57 of 59 patients (96.6\%). In the remaining two patients, whose indication for ERCP was cholangitis associated with acute pancreatitis, EBS was not performed and a biliary plastic stent was placed. Sphincteroplasty was performed in 15 of the 57 patients that underwent EBS. None of the two patients in whom EBS was not performed received sphincteroplasty. Biliary sludge or stones were found and successfully removed in most patients (73.3\%). In eight patients (13.6\%) a biliary plastic stent was placed either to facilitate the removal of lithiasic material or to treat or prevent acute cholangitis. With the aim of preventing post-ERCP acute pancreatitis, a pancreatic plastic stent was placed in 3 subjects (5.1\%).
ERCP-related complications were reported in 9 patients (15.3\%). The most frequent complication was acute pancreatitis (6.8\%). Bleeding occurred in two patients $(3.4 \%)$ who were not under antiplatelet or anticoagulant treatment. Perforation was diagnosed in two cases (3.4\%). Post-ERCP cholangitis was reported in a single patient (1.7\%). All complications were mild and conservatively managed except for one patient with severe pancreatitis who died after ERCP perforation.

\subsection{Impact of biliary sphincterotomy at the index admission}

Baseline characteristics of patients who underwent EBS (23\%, $57 / 247)$ and patients who did not receive EBS $(77 \%, 190 / 247)$ at the index admission are displayed in Table 2 . The two groups were comparable regarding age (there were statistically significant differences that were not clinically relevant) and comorbidity. Altered liver biochemistry at the time of admission was significantly more frequent in the EBS group compared with the no EBS arm (56/57, $98.2 \%$ vs. $153 / 190,80.5 \%$; $=0.01$ ). As expected, patients in the group that underwent EBS had a significantly higher rate of confirmed choledocholithiasis on image tests ( $66 \% \mathrm{vs.} 31 \%, \mathrm{p}=0.0001$ ). There were no differences between both groups regarding the severity of AGP, length of stay, and the use of UDCA after hospital discharge.

Globally, recurrence of AGP occurred in 17.4\% (43/247) patients. The cumulative one-year incidence of a new episode of AGP in patients with EBS was $1.8 \%$ (95\% CI: $0.2-12 \%$ ), significantly lower than the incidence found in patients who did not undergo EBS (23\%; 95\% CI: $17-31 \%)(\mathrm{p}=0.006)$ as shown in Fig. 2. In the Cox proportional-hazards model, EBS was independently associated with a lower recurrence of AGP (HR 0.29, 95\% CI: 0.08-0.92, $\mathrm{p}=0.037$ ) (Table 3 ). Moreover, EBS was associated with a decrease in all gallstone-related readmissions (HR 0.46, 95\% CI: 0.21-0.98, 
Table 1

Characteristics of the study population.

\begin{tabular}{ll}
\hline & $\mathrm{n}=247$ \\
\hline Age, mean \pm SD (years) & $80.3 \pm 12.6$ \\
Sex & \\
Male & $101(40.9 \%)$ \\
Female & $146(59.1 \%)$ \\
ASA & \\
I & $5(2 \%)$ \\
II & $13(5.3 \%)$ \\
III & $191(77.3 \%)$ \\
IV & $38(15.4 \%)$ \\
Charlson index, median (IQR) & $5(4-6)$ \\
Hypertension & $171(69.2 \%)$ \\
Diabetes mellitus & $51(20.5 \%)$ \\
Ischemic cardiopathy & $43(17.4 \%)$ \\
Congestive heart disease & $46(18.4 \%)$ \\
Peripheral vascular disease & $23(9.3 \%)$ \\
Cerebrovascular disease & $30(12.5 \%)$ \\
Hemiplegia & $1(0.4 \%)$ \\
Dementia & $36(14.7 \%)$ \\
Moderate to severe CKD & $18(7.2 \%)$ \\
Moderate to severe CLD & $6(2.4 \%)$ \\
Connective tissue disease & $4(1.6 \%)$ \\
Peptic ulcer disease & $9(3.6 \%)$ \\
COPD & $25(10.1 \%)$ \\
Hematologic disease (leukemia, lymphoma) & $5(2 \%)$ \\
Localized solid tumor & $21(8.5 \%)$ \\
Metastatic solid tumor & $4(1.6 \%)$ \\
AIDS & $1(0.4 \%)$ \\
Severity of pancreatitis & \\
Mild & $178(72 \%)$ \\
Moderate & $38(15.4 \%)$ \\
Severe & $31(12.6 \%)$ \\
ChU admission & $16(6.4 \%)$ \\
Ultrasonography & $97(39.2 \%)$ \\
CT & $43(44.3 \%)$ \\
MRI & $10(10.3 \%)$ \\
ERCP & $25(25.8 \%)$ \\
\hline C & $19(19.6 \%)$ \\
Cegorical & $59(23.9 \%)$ \\
\hline
\end{tabular}

Categorical variables are described as absolute and relative frequencies. SD: standard deviation; ASA: American Society of Anesthesiologist classification; IQR: interquartile range; CKD: chronic kidney disease (moderate=creatinine level $>3 \mathrm{mg} / \mathrm{dL}$; severe = dialysis, status post kidney transplant or uremia); CLD: chronic liver disease (moderate: cirrhosis and portal hypertension without variceal bleeding history; severe: cirrhosis and portal hypertension with variceal bleeding history); COPD: chronic obstructive pulmonary disease; AIDS: acquired immune deficiency syndrome; ICU: intensive care unit; ERCP: endoscopic retrograde cholangiopancreatography.

Table 2

Baseline characteristics of patients who did and did not undergo endoscopic biliary sphincterotomy.

\begin{tabular}{llll}
\hline & EBS $=57$ & No EBS $=190$ & $p$ \\
\hline $\begin{array}{l}\text { Age, mean } \pm \text { SD (years) } \\
\text { Sex }\end{array}$ & $82.9 \pm 8.2$ & $79.5 \pm 13.6$ & 0.02 \\
$\quad$ Male & $25(43.9 \%)$ & $76(40 \%)$ & 0.6 \\
$\quad$ Female & $32(56.1 \%)$ & $114(60 \%)$ & \\
Comorbidity & & & 0.45 \\
ASA & $2(3.5 \%)$ & $3(1.6 \%)$ & \\
I & $5(8.8 \%)$ & $8(4.2 \%)$ & \\
II & $42(73.7 \%)$ & $149(78.4 \%)$ & \\
III & $8(14 \%)$ & $30(15.8 \%)$ & 0.2 \\
IV & $5.4 \pm 1.8$ & $4.8 \pm 1.7$ & 0.1 \\
Charlson index, mean \pm SD & & & \\
Pancreatitis severity, No. (\%) & $40(70.2 \%)$ & $138(72.7 \%)$ & \\
$\quad$ Mild & $13(22.8 \%)$ & $25(13.1 \%)$ & \\
$\quad$ Moderate & $4(7 \%)$ & $27(14.2 \%)$ & \multirow{2}{*}{0.0001} \\
$\quad$ Severe & $38(66 \%)$ & $59(31 \%)$ & 0.26 \\
Choledocholithiasis on image test & $17.4 \pm 16.8$ & $13.6 \pm 23.3$ & 0.43 \\
Length of stay, mean \pm SD (days) & $14(24.5 \%)$ & $36(18.9 \%)$ & \\
UDCA at discharge & &
\end{tabular}

Categorical variables were described as absolute and relative frequencies. EBS: endoscopic biliary sphincterotomy; SD: standard deviation; IQR: interquartile range; ASA: American Society of Anesthesiologist classification; UDCA: ursodeoxycholic acid.
A
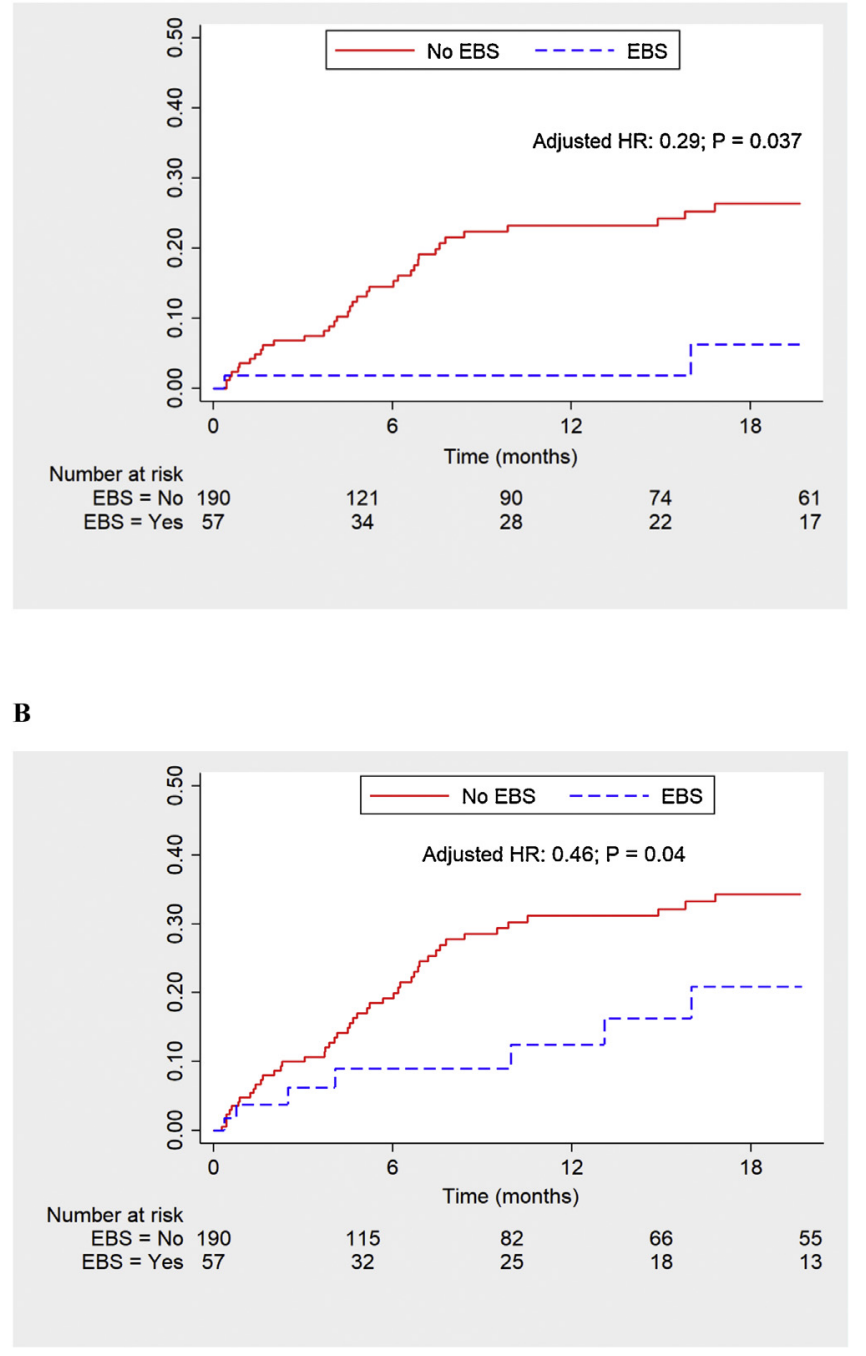

Fig. 2. (A) Risk of acute gallstone pancreatitis recurrence. (B) Risk of biliary-related disease recurrence.

EBS: endoscopic biliary sphincterotomy.

$\mathrm{p}=0.043$ ) (Fig. 2). The number of EBS needed to prevent one AGP was 6.

\subsection{Follow-up}

Median time of follow up was 426 days (IQR 85-916). Twentysix percent (64/247) of patients had further admissions for gallstone-related disease: 51 patients $(20.6 \%)$ were readmitted once, 7 patients twice (2.8\%), one patient three times, and 5 patients $\geq 4$ times. Median time to first readmission for AGP was 8 months (IQR 1.9-24.6); median time to first readmission for a biliary-related disease was 7 months (IQR 1.7-21.6). During first readmission 12 patients underwent EBS, so that a total of 69 patients received EBS before second readmission ( 57 patients during index admission and 12 patients during first readmission). Detailed data of the first three readmissions are outlined in Table 4.

\subsection{Mortality}

Overall one-year survival was $82.5 \%$ (76.5-87.1\%). At the end of follow-up 59 out of the initial 247 (23.8\%) patients had died: $32(54.2 \%)$ were nonbiliary-related deaths and 27 (45.8\%) were 
Table 3

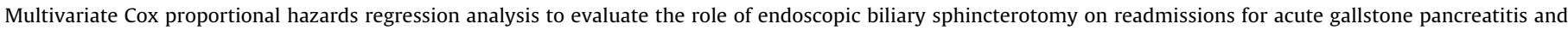
biliary-related disease and for overall survival.

\begin{tabular}{|c|c|c|c|c|c|c|}
\hline & \multicolumn{3}{|c|}{ AGP recurrence } & \multicolumn{3}{|c|}{ Any gallstone-related event } \\
\hline & Adjusted HR & $95 \% \mathrm{CI}$ & $p$ & Adjusted HR & $95 \% \mathrm{CI}$ & $p$ \\
\hline EBS & 0.29 & $0.08-0.92$ & 0.037 & 0.46 & $0.21-0.98$ & 0.043 \\
\hline Age & 1.03 & $1.00-1.06$ & 0.06 & 1.03 & $1.00-1.06$ & 0.045 \\
\hline Choledocholithiasis & 0.38 & $0.10-0.88$ & 0.02 & 0.87 & $0.49-1.56$ & 0.65 \\
\hline \multicolumn{7}{|l|}{ Pancreatitis severity } \\
\hline Mild & Reference & & & Reference & & \\
\hline Moderate & 1.17 & $0.52-2.7$ & 0.70 & 1.11 & $0.56-2.23$ & 0.76 \\
\hline Severe & 0.44 & $0.11-1.8$ & 0.26 & 0.74 & $0.27-2.07$ & 0.57 \\
\hline UDCA & 0.99 & $0.4-2.1$ & 0.99 & 1.21 & $0.67-2.18$ & 0.53 \\
\hline
\end{tabular}

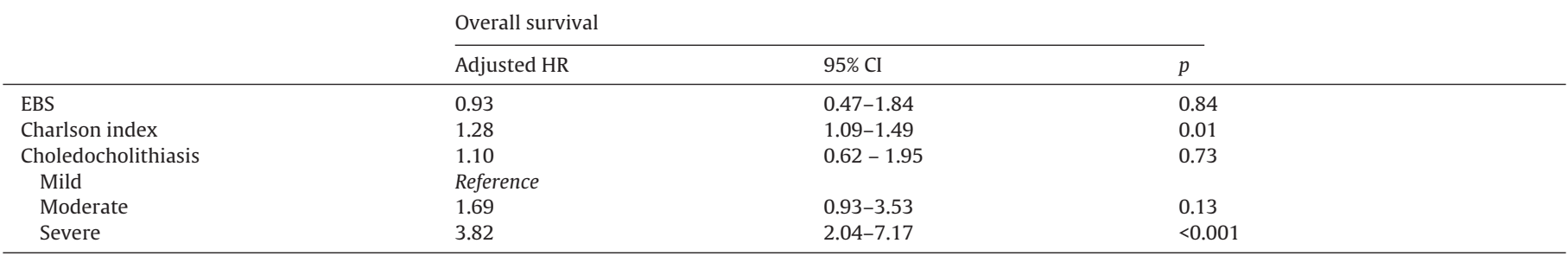

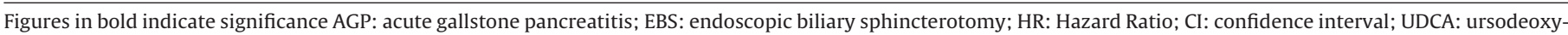
cholic acid.

Table 4

Characteristics of the first three readmissions for biliary-related disease.

\begin{tabular}{|c|c|c|c|}
\hline Number of patients readmitted & $\begin{array}{l}\text { First readmission } \\
64\end{array}$ & $\begin{array}{l}\text { Second readmission } \\
13\end{array}$ & $\begin{array}{l}\text { Third readmission } \\
6\end{array}$ \\
\hline \multicolumn{4}{|l|}{ Reason for readmission } \\
\hline Acute pancreatitis & $40(62.5 \%)$ & $10(77 \%)$ & $4(66.6 \%)$ \\
\hline Cholecystitis & $16(25 \%)$ & $2(15.3 \%)$ & $1(16.7 \%)$ \\
\hline Cholangitis & $6(9.4 \%)$ & $1(7.7 \%)$ & 0 \\
\hline Choledocholithiasis & $2(3.1 \%)$ & 0 & $1(16.7 \%)$ \\
\hline EBS in previous admissions & $8(12.5 \%)$ & $3(23 \%)$ & $2(33 \%)$ \\
\hline Index admission & 8 & 2 & 0 \\
\hline First readmission & & 1 & 0 \\
\hline Second readmission & & & 2 \\
\hline ERCP performed in current readmission & $13(20 \%)$ & $4(30 \%)$ & $2(33 \%)$ \\
\hline \multicolumn{4}{|l|}{ Indication for ERCP } \\
\hline Confirmed/suspected choledocholithiasis & 9 & 3 & 0 \\
\hline Acute cholangitis & 4 & 1 & 1 \\
\hline Preventive intention & 0 & 0 & 1 \\
\hline
\end{tabular}

EBS: endoscopic biliary sphincterotomy; ERCP: endoscopic retrograde cholangiopancreatography.

attributed to gallstone-related disease. In-hospital mortality during first admission was 8\% (20/247). Of the 20 patients that died during first admission, three presented moderate pancreatitis and seven had severe pancreatitis (three of which died from pancreatitis in spite of successful EBS). Of the 227 patients who survived index admission, 188 patients were alive at the end of follow-up and 39 died during follow-up ( 7 due to biliary disease and 32 because of non-biliary causes). Of the seven patients who died due to biliary disease, six died during first readmission (four acute pancreatitis, two cholecystitis) and one died during second readmission.

\section{Discussion}

Patients who do not undergo cholecystectomy after a first episode of AGP present a remarkably high recurrence of pancreatitis $[4,8,26]$. Moreover, these patients are also at increased risk of other gallstone-related complications such as acute cholecystitis, choledocholithiasis, and cholangitis [27-30]. Our study reasserts this known data, with $26 \%$ of patients being readmitted for gallstone-related disorders during follow-up. Furthermore, it shows that EBS reduces the risk of AGP recurrence and other biliary-related disorders in this context.
Due to this high risk of recurrent disease, prompt cholecystectomy during the initial admission after a first episode of AGP is widely recommended in order to prevent further episodes of gallstone disorders [15,31-33]. The increase in life expectancy along with the higher prevalence of gallstone-related disorders with advanced age are leading to a significant rise of AGP in frail and elderly patients. In consequence, patients who are unfit for surgery are becoming frequently encountered in clinical practice and elective cholecystectomy after an episode of AGP is often dismissed [17]. Most of these subjects do not receive any preventive treatment during hospital admission or after discharge with a consequent high readmission rate. For this reason, other prophylactic measures need to be explored. To date, some of these non-surgical approaches, such as UDCA treatment, have been tested without consistent results [34,35]. Recently, EBS has been advocated as an alternative to cholecystectomy in this population $[4,13,17]$.

We identified 636 patients with gallbladder in situ admitted for a first episode of AGP. Of them, 247 patients (38.8\%) were deemed not fit for elective surgery, a figure similar to that observed in a recent report [4]. Even though no specific criteria were followed to dismiss patients for cholecystectomy, advanced age or high comorbidity were the reasons employed by the physicians in charge. 
We evaluated the benefit of ERCP with EBS in this selected cohort of patients and excluded those who underwent ERCP without EBS, as previous studies have shown that nontherapeutic ERCP has no benefit on readmission rates compared with no intervention [4]. Unlike previous reports [22], we did not exclude from the analysis patients with severe pancreatitis to limit selection bias. During the first year of follow-up, a significantly lower rate of recurrence of AGP was observed in EBS group. Even though most episodes of recurrent AGP when the gallbladder is left in place occur within the first months after the index attack [27], we observed that both groups continued to be at risk for recurrent AGP years after the initial episode. In consonance with our results, a recent study showed that ERCP in patients who did not undergo cholecystectomy significantly reduced the risk of recurrent pancreatitis [13]. Similarly, Vazquez-lglesias et al. [22] in a single-arm prospective study followed 88 patients with AGP and an intact gallbladder who underwent EBS because of high surgical risk. They reported that only $2.2 \%$ of patients experienced recurrent pancreatitis. EBS may prevent biliary events by facilitating passage of bile duct stones into the duodenum, modifying bile lithogenicity, and thus inhibiting further formation of gallstones [19,22,36,37]. This could explain the lower rate of biliary events other than pancreatitis in our cohort and previous reports. Even though EBS significantly reduced AGP recurrence, there were some patients who presented new attacks. Most of these recurrences are probably related to inadequate EBS or the development of fibrosis after sphincterotomy, as suggested in some studies [19,22].

The main indications to perform ERCP were the presence of concurrent cholangitis or evidence of biliary obstruction, as they are widely recognized indications for ERCP in the acute pancreatitis setting [2]. ERCP was indicated with a preventive intention in $3.4 \%$ of our cohort. This finding was expected considering that the role of ERCP intended only to prevent new episodes of AGP in patients who do not undergo cholecystectomy is less clear. Therefore, the presence of choledocholithiasis was predictably higher in the EBS group. Interestingly, EBS remained as a protective therapy after adjusting by the presence of choledocholithiasis in the multivariate analysis. Even if UDCA is not a proven prophylactic therapy, EBS showed a protective role after adjusting by UDCA use.

The time for ERCP remains controversial. ERCP was performed during hospital admission in almost $80 \%$ of cases. Contrary to other studies that found a longer length of hospitalization in patients who underwent ERCP [13], the length of stay in the EBS group was similar. Even though the most appropriate time for EBS when performed prophylactically is unclear, it seems reasonable to perform ERCP during admission before hospital discharge, both for the comfort of the patient and from a cost-benefit perspective [38].

The rate of ERCP-related complications was $15.3 \%$. Reported ERCP complications rates vary in the literature due to the heterogeneity in patient populations, study designs and definitions. Post-ERCP pancreatitis in our cohort of elderly patients with significant comorbidity was $6.8 \%$, within published rates (1.6-15.7\%) [16]. Even though ERCP is an invasive technique that may associate complications, the alternative of not performing preventive measures leads to a high risk of pancreatitis recurrence, a potentially life-threatening condition with elevated morbidity especially in elderly and comorbid patients.

We believe that our results have relevant clinical implications for patients with AGP who are unfit for surgery, as they contribute with additional evidence regarding the protective role of EBS. However, we acknowledge that this study has some limitations given its retrospective nature and taking into account that no specific criteria were followed to consider a patient to be at high risk for surgery. Besides, it is also possible that the recurrence rate may be underestimated, as we assumed that if there was no documented evidence of pancreatitis in the medical records, then recurrence did not occur.
In summary, recurrence of AGP is frequent if cholecystectomy is not performed. Our study suggests that EBS could be considered as a preventive measure after a first episode of AGP when elective cholecystectomy is not an option in elderly patients or with significant comorbidity. Nevertheless, further prospective randomized studies are needed to define the best strategy in the increasing population of patients who are unfit for surgery.

\section{Conflict of interest}

None declared.

\section{References}

[1] Yadav D, Lowenfels AB. The epidemiology of pancreatitis and pancreatic cancer Gastroenterology 2013;144:1252-61.

[2] Working Group IAP/APA Acute Pancreatitis Guidelines. IAP/APA evidencebased guidelines for the management of acute pancreatitis. Pancreatology 2013:13:1-15.

[3] Acosta JM, Ledesma CL. Gallstone migration as a cause of acute pancreatitis. N Engl J Med 1974;290:484-7.

[4] Qayed E, Shah R, Haddad YK. Endoscopic retrograde cholangiopancreatography decreases all-cause and pancreatitis readmissions in patients with acute gallstone pancreatitis who do not undergo cholecystectomy: a nationwide 5-year analysis. Pancreas 2018;47(4):425-35.

[5] Hernandez V, Pascual I, Almela P, Añón R, Herreros B, Sanchiz V, et al. Recurrence of acute gallstone pancreatitis and relationship with cholecystectomy or endoscopic sphincterotomy. Am J Gastroenterol 2004;99(12):2417-23.

[6] Ranson JH. The role of surgery in the management of acute pancreatitis. Ann Surg 1990;211(4):382-93.

[7] Cameron DR, Goodman AJ. Delayed cholecystectomy for gallstone pancreatitis: readmissions and outcomes. Ann R Coll Surg Engl 2004;86(5):358-62.

[8] Trust MD, Sheffield KM, Boyd CA, Benarroch-Gampel J, Zhang D, Townsed Jr $\mathrm{CM}$, et al. Gallstone pancreatitis in older patients: are we operating enough? Surgery 2011;150(3):515-25.

[9] Berlinski LS, Dorazio RA, Winkley JH. Gallstone pancreatitis. Sinai J Med 1979;46:364-6.

[10] Howard JM, Ehrlich EW. Gallstone pancreatitis: a clinical entity. Surgery 1962;51:177-83.

[11] Kelly TR. Gallstone pancreatitis: pathophysiology. Surgery 1976;80:488-92

[12] Moreau JA, Zinsmeister AR, Melton LJ, DiMagno EP. Gallstone pancreatitis and the effect of cholecystectomy: a population-based cohort study. Mayo Clin Proc 1988;63:466-73

[13] Hwang SS, Li BH, Haigh PI. Gallstone pancreatitis without cholecystectomy JAMA Surg 2013;148(9):867-72.

[14] Kaw M, Al-Antably Y, Kaw P. Management of gallstone pancreatitis: cholecystectomy or ERCP and endoscopic sphincterotomy. Gastrointest Endosc 2002;56(1):61-5.

[15] Lankisch PG, Apte M, Banks PA. Acute pancreatitis. Lancet 2015;386:85-96.

[16] Committee, ASGE Standards of Practice. The role of endoscopy in benign pancreatic disease. Gastrointest Endosc 2015;82(2):203-14.

[17] Bignell M, Dearing M, Hindmarsh A, Rhodes M. ERCP and endoscopic sphincterotomy (ES): a safe and definitive management of gallstone pancreatitis with the gallbladder left in situ. J Gastrointest Surg 2011;15(12):2205-10.

[18] Hammarstrom LE, Stridbeck H, Ihse I. Effect of endoscopic sphincterotomy and interval cholecystectomy on late outcome after gallstone pancreatitis. Br J Surg 1998;85:333-6.

[19] Welbourne CR, Beckley DE, Eyre-Brook IA. Endoscopic sphincterotomy without cholecystectomy for gallstone pancreatitis. Gut 1995;37:119-20.

[20] Lee J, Ryu J, Park J, Yoon WJ, Lee SH, Hwang JH, et al. Roles of endoscopic sphincterotomy and cholecystectomy in acute biliary pancreatitis. Hepatogastroenterology 2008;55:1981-5.

[21] Working Party of the British Society of Gastroenterology, Association of Surgeons of Great Britain and Ireland, Pancreatic Society of Great Britain and Ireland, Association of Upper GI Surgeons of Great Britain and Ireland. UK guidelines for the management of acute pancreatitis. Gastroenterology 2005;54(Suppl 3):iii1-9.

[22] Vázquez-lglesias JL, González-Conde B, López-Rosés L, Estévez-Prieto E, Alonso-Aguirre P, Lancho A, et al. Endoscopic sphincterotomy for prevention of the recurrence of acute biliary pancreatitis in patients with gallbladder in situ. Surg Endosc 2004; 18:1442-6.

[23] Tenner S, Baillie J, De Witt J, Vege SS, American College of Gastroenterology. American College of gastroenterology guideline:management of acute pancreatitis. Am J Gastroenterol 2013;108:1400-15.

[24] Cotton PB, Eisen GM, Aabakken L, Baron TH, Hutter MM, Jacobson BC, et al. A lexicon for endoscopic adverse events: report of an ASGE workshop. Gastrointest Endosc 2010;71(3):446-54.

[25] Altman DG, Andersen PK. Calculating the number needed to treat for trials where the outcome is time to an event. BMJ 1999;319(7223):1492-5.

[26] Green R, Charman SC, Palser T. Early definitive treatment rate as a quality indicator of care in acute gallstone pancreatitis. Br J Surg 2017;104:1686-94. 
[27] van Baal MC, Besselink MG, Bakker OJ, van Santvoort HC, Schaapeherder AF, Nieuwenhuijs VB, et al. Timing of cholecystectomy after mild biliary pancreatitis: a systematic review. Ann Surg 2012;255(5):860-6.

[28] Ito K, Ito H, Whang EE. Timing of cholecystectomy for biliary pancreatitis: do the data support current guidelines? J Gastrointest Surg 2008;12(12):2164-70.

[29] Sanjay P, Yeeting S, Whigham C, Judson H, Polignano FM, Tait IS. Endoscopic sphincterotomy and interval cholecystectomy are reasonable alternatives to index cholecystectomy in severe acute gallstone pancreatitis. Surg Endosc 2008;22(8):1832-7.

[30] Heider TR, Brown A, Grimm IS, Behrns KE. Endoscopic sphincterotomy permits interval laparoscopic cholecystectomy in patients with moderately severe gallstone pancreatitis. J Gastrointest Surg 2006;10(1):1-5.

[31] McMahon A, Fischbacher C, Frame S, MacLeod MC. Impact of laparoscopic cholecystectomy: a population-based study. Lancet 2000;356:1632-7.

[32] Kimura Y, Takada T, Kawarada Y, Hirata K, Mayumi T, Yoshida M, et al. JPN guidelines for the management of acute pancreatitis: treatment of gallstoneinduced acute pancreatitis. J Hepatobiliary Pancreat Surg 2006;13(1):56-60.
[33] Wilson CT, de Moya MA. Cholecystectomy for acute gallstone pancreatitis: early vs delayed approach. Scand J Surg 2010;99(2):81-5.

[34] Ros E, Navarro S, Bru C, Garcia-Pugés A, Valderrama R. Occult microlithiasis in ééidiopathic' acute pancreatitis: prevention of relapses by cholecystectomy or ursodeoxycholic acid therapy. Gastroenterology 1991;101:1701-9.

[35] Testoni PA, Caporuscio S, Bagnolo F, Lella F. Idiopathic reCurrent pancreatitis: long-term results after ERCP, endoscopic sphincterotomy, or ursodeoxycholic acid treatment. Am J Gastroenterol 2000;95:1702-7.

[36] Siegel JH, Veerappan A, Cohen SA, Kasmin FE. Endoscopic sphincterotomy for biliary pancreatitis: an alternative to cholecystectomy in high-risk patients. Gastrointest Endosc 1994;40:573-5.

[37] Hutton SW, Sievert CE, Vennes JA, Duane WC. The effect of sphincterotomy on gallstone formation in the prairie dog. Gastroenterology 1981;81:663-7.

[38] Gislason H, Vetrhus M, Horn A, Hoem D, Söndenaa K, Soreide O, et al. Endoscopic sphincterotomy in acute gallstone pancreatitis: a prospective study of the late outcome. Eur J Surg 2001;167(3):204-8. 